\title{
La comparaison épistémique en français moderne
}

\author{
Catherine Fuchs \\ LATTICE (CNRS/ENS) \\ catherine.fuchs@ens.fr
}

\section{Introduction}

La comparaison dite 'épistémique' (Rivara, 1979: ch. X; et 1990 : 100, note 13) se distingue de la comparaison quantitative ordinaire (que nous appellerons 'standard') en ce qu'elle opère au niveau 'métalinguistique' et non pas au niveau prédicatif (Muller, 1996 : 136-138; Amsili \& Desmets, sous presse : $§ 2$ ). Alors que la comparaison standard instaure une égalité ou une inégalité de degré à propos de la qualité prédiquée des entités, la comparaison épistémique instaure une égalité ou une inégalité de degré à propos de l'assertion des deux contenus propositionnels (plus précisément, à propos de leur adéquation au réel, de leur exactitude, de leur pertinence) :

Son geste était plus irréfléchi que son discours (standard)

"L’irréflexion de son geste était plus grande que celle de son discours"

Son geste était plus irréfléchi que méchant (épistémique)

"Il est plus exact (plus juste, plus pertinent) de dire que son geste était irréfléchi que de dire qu'il était méchant"

Alors que la comparaison standard prédique le plus souvent une même qualité à propos de deux entités, la comparaison épistémique met en jeu deux qualités, généralement prédiquées d'une même entité, parfois distribuées sur deux entités :

Son geste était moins irréfléchi que ses propos déplacés (épistémique)

"Il est moins exact (moins juste, moins pertinent) de dire que son geste était irréfléchi que de dire que ses propos étaient déplacés"

Il arrive toutefois qu'une lecture standard s'applique à un schéma mettant en jeu deux qualités ${ }^{1}$ et, à l'inverse, qu'une lecture épistémique s'applique à un schéma mettant en jeu une seule qualité :

Le siège, placé au-dessus du plancher de toute la longueur des jambes de l'enfant, sera aussi large que les cuisses sont longues (décret du 25 octobre 1894 ; cit. Damourette \& Pichon) (standard)

"la largeur du siège sera égale à la longueur des cuisses"

Le français, pas plus que l'anglais, n'est la langue de communication que l'on parle en Afrique de l'Ouest (ex. de Muller, 1996 : 138) (épistémique)

"Il n'est pas plus exact de dire que l'anglais est la langue ... qu'il n'est exact de dire que le français est la langue ..."

Par ailleurs, les cas de double lecture d'un même schéma sont loin d'être rares.

Si la comparaison épistémique a été étudiée de façon approfondie sur l'anglais (Rivara : 1979) ${ }^{2}$, tel n'est pas le cas concernant le français - en tout cas le français moderne ${ }^{3}$. La présente étude vise à combler ce manque. Il s'agira, en s'appuyant au maximum sur des exemples attestés (du $18^{\mathrm{e}}$ siècle à nos jours), de dégager les conditions (syntaxiques, sémantiques et pragmatiques) de la lecture épistémique des schémas comparatifs et de caractériser les cas d'incertitude interprétative.

\section{Les indices formels}

Pour identifier les indices formels de la comparaison épistémique, on examinera successivement les marqueurs comparatifs, puis la structure de la phrase comparative. 


\subsection{Les marqueurs comparatifs}

\subsubsection{Les quatre types de marqueurs spécifiques à la comparaison épistémique}

a) le marqueur autant postposé à un adjectif :

Il est modeste autant qu'habile (Ac., 1835 ; cit. TLF) (épistémique)

vs. Il est aussi modeste que son frère (standard)

b) le comparatif non synthétique :

Le bon vendeur est plus bon à écouter qu'à parler (Google) (épistémique)

vs. Il est meilleur à la belote qu'aux échecs (standard)

c) les marqueurs plutôt (alternant avec plus) et aussi bien (alternant avec autant) :

Il est plus/plutôt grincheux que méchant (épistémique)

C'était une passion innocente autant/aussi bien qu'extravagante (épistémique)

d) le marqueur pas tant pour nier l'égalité :

Il n'est pas tant méchant que grincheux (épistémique)

vs. Il n'est pas si/aussi méchant que son frère (standard)

\subsubsection{Les marqueurs non spécifiques}

En dehors de ces quelques indices spécifiques, tous les autres marqueurs comparatifs sont neutres quant aux lectures épistémique ou standard. C'est le cas de aussi, plus, moins, pas aussi, pas plus, pas moins placés devant l'adjectif, même si certaines indications (liaisons et pauses) peuvent exister à l'oral :
il est pluS \# idiot que méchant (épistémique)
vs. Il est pluZ-idiot que son frère (standard)
Il est idiot, plu(s) que méchant (épistémique)
vs. Il est idiot, pluS que son frère (standard)

C'est également le cas de ces mêmes marqueurs et de autant employés avec des verbes :

L'employée ne rit pas plus qu'elle ne lève les épaules (Le Monde) (ex. de Muller, 1996 : 138:

« seul le sens permet de trancher en faveur de l'épistémique »)

On y [au Café de Montmartre] achète autant qu'on y boit (Fargue) (standard ou épistémique)

En présence de compléments, la place (antéposée ou postposée) du marqueur peut toutefois constituer un indice :

Il tient de sa mère autant que de son père (favorise plutôt la lecture épistémique)

vs. Il tient autant de sa mère que de son père (favorise plutôt la lecture standard)

\subsubsection{Adverbes}

Dans certains cas, la présence d'adverbes oriente l'interprétation. La lecture épistémique se trouve ainsi exclue en présence de modifieurs quantitatifs qui jouent sur le caractère gradable des adjectifs (encore, $\grave{a}$ peine, guère, un peu, beaucoup, bien, infiniment, ...), alors qu'elle s'imposera au contraire avec un adverbe qualitatif qui joue sur la vérité de l'assertion (vraiment, peut-être, ...) :

Je suis convaincu (...) que cette mort est infiniment plus douce qu'elle n'est cruelle (Sade) (standard)

Je ne pus jamais le décider à venir voir ce spectacle qu'il s'obstinait à trouver encore plus triste 
que curieux (de Boigne) (standard)

On était plus indignés que vraiment surpris (France-Inter) (épistémique)

(...); en rapport à quoi actions et révolutions sont moins des catalyseurs peut-être que simplement des marqueurs. (Jullien) (épistémique)

\subsection{La structure de la phrase comparative}

\subsubsection{La mobilité}

La mobilité (déplaçabilité et détachabilité) du marqueur comparatif suivi de la subordonnée est caractéristique de la comparaison épistémique :

Le bon vendeur est plus bon à écouter qu'à parler; Le bon vendeur est bon à écouter, plus qu'à parler; Plus qu'à parler, le bon vendeur est bon à écouter

Il est plus/plutôt grincheux que méchant ; Il est grincheux, plus/plutôt que méchant ; Plus/plutôt que méchant, il est grincheux.

Une telle segmentation de la phrase pousse en effet à assigner une portée extraprédicative au quantificateur; et donc à y voir, non pas une mesure du degré des qualités (lecture standard), mais une mesure du degré d'adéquation au réel des assertions (d'où une lecture épistémique). Par cette dissociation entre la prédication et l'évaluation du degré, se trouve ainsi favorisée l'interprétation de la comparative comme une 'construction à parallélisme', càd. comme une « comparative portant sur deux phrases » (Muller, $1996: 136 s q$.).

Néanmoins, la lecture épistémique n'est pas la seule possible. En effet, toute comparative peut être segmentée, même en lecture standard; le segment détaché à droite fonctionnant alors comme un ajout après coup, et le segment détaché à gauche comme l'annonce d'un élément focalisé :

Il aime son confort, plus encore que sa famille

"Il aime son confort, (et j'ajoute qu')il l'aime plus encore que sa famille"

Plus encore que sa famille, il aime son confort

"(Il y a quelque chose) plus encore que sa famille, (qu)'il aime ( : c'est) son confort"

\subsubsection{La subordonnée}

Le caractère réduit ou non de la subordonnée ne constitue pas un indice totalement fiable en français ${ }^{4}$, même si la comparaison épistémique va souvent de pair avec une subordonnée averbale, et la comparaison standard avec une subordonnée verbale :

Monsieur de Marcellus, plus bon que méchant dans le fond, profita mal du conseil (de Boigne) (épistémique)

(...) le duc de Montpensier, aussi bon, aussi aimable, aussi gracieux qu'il était distingué, mourut d'une maladie étrange (de Boigne) (standard)

\subsubsection{Négation et items de polarité négative}

Une négation présente dans la matrice aura une portée intrapropositionnelle en cas d'interprétation standard, et une portée métalinguistique dans l'interprétation épistémique :

Ce fruit n'est pas aussi bon qu'il est beau (standard)

Je ne parlerai pas plus de la désastreuse retraite de Moscou que des glorieuses campagnes qui l'avaient précédée (de Boigne) (épistémique) 
Dans la subordonnée, la présence d'une négation, impossible avec la lecture standard, est au contraire possible avec la lecture épistémique :

* Ce trou est plus profond qu'il n'est pas large; * Ce trou est plus profond que pas large (standard)

Jean est plus/plutôt prudent que pas courageux (épistémique)

A l'inverse, les items de polarité négative sont acceptés dans la subordonnée avec la lecture standard, mais pas avec la lecture épistémique :

Je suis plus fâché envers toi que je ne l'ai jamais été envers quiconque (standard)

* Il est plutôt grincheux que méchant envers n'importe qui (épistémique)

\section{L'interprétation sémantique}

Le type d'interprétation (épistémique ou standard) d'un schéma comparatif dépend en partie du sémantisme des prédicats. Néanmoins, les cas d'incertitude interprétative restent nombreux.

\subsection{Le sémantisme des prédicats}

\subsubsection{La gradabilité}

L'un des indices les plus fiables de l'épistémique est la présence de qualités non gradables :

Nous sommes automates autant qu'esprits (Barrès)

Celui-là aussi a son acte de décès légal autant que britannique (Bloy)

J'étais plus libérale que bourbonnienne (de Boigne)

Vous qui répondez, vous, moins juge qu'assassin, vous qui de Jean Calas avez proscrit la tête (...) (Chénier)

Ainsi, lorsque des adjectifs antonymes 'polaires' ou 'complémentaires', c'est-à-dire non gradables (marquant une relation logique de contradiction), figurent dans un schéma comparatif, celui-ci s'interprète comme de l'épistémique : c'est le cas de l'expression plus mort que vif, qui équivaut littéralement à "plutôt mort que vivant".

A l'inverse, lorsque les prédicats renvoient à des qualités dont le caractère gradable est objectivement mesurable, l'interprétation standard tend à s'imposer aux dépens de l'épistémique. Tel est le cas, notamment avec des adjectifs 'dimensionnels' (par différence avec les adjectifs 'appréciatifs' ; cf. Klein : 1991) :

La main de Paul est aussi large que longue

Le puits est plus profond que l'échelle n'est haute

En l'absence de telles indications, c'est le parallélisme sémantique entre les deux contenus propositionnels qui induit l'interprétation épistémique.

\subsubsection{Mise en facteur commun du prédicat}

Un premier type de parallélisme consiste en la mise en facteur commun d'un même prédicat en relation avec deux entités sémantiquement opposées (par exemple deux espèces d'un même genre); l'égalité ou l'inégalité porte alors sur les degrés relatifs d'exactitude qu'il peut y avoir à asserter chacune des propositions, càd. à attribuer le prédicat à l'une ou l'autre entité : 
Ce qu'on ignore, c'est que la tortue terrestre, aussi bien que la tortue aquatique, inspire une grande répugnance au peuple grec (About)

"dire de la tortue terrestre qu'elle inspire une grande répugnance au peuple grec est aussi exact que de le dire de la tortue aquatique"

Ce sont les transformations silencieuses plus que la force des Masses insurgées (...) qui renversent et renverseront tous les Anciens Régimes (Jullien)

"dire des transformations silencieuses que ce sont elles qui renversent et renverseront tous les Anciens Régimes est plus exact que de le dire de la force des Masses insurgées"

\subsubsection{Mise en facteur commun de l'entité}

Le second type de parallélisme (beaucoup plus courant) consiste, à l'inverse, en la mise en facteur commun d'une même entité en relation avec deux qualités entretenant une relation sémantique étroite (de synonymie ou d'antonymie ${ }^{5}$ ). Seul le schéma d'inégalité est alors attesté en lecture épistémique ${ }^{6}$. Il exprime la différence de degré d'exactitude qu'il peut y avoir à prédiquer l'une ou l'autre qualité à propos de l'entité. On parlera de 'parallélisme par symétrie' dans le cas de deux qualités parasynonymes :

Ils s'étaient moins corrompus qu'avilis (Rousseau)

"d'eux, il est moins exact de dire qu'ils s'étaient corrompus que de dire qu'ils s'étaient avilis", d'où : "moins (vraiment) corrompus que (plutôt) avilis"

Le deuil encore récent pour la princesse Charlotte ne permettait pas les plaisirs bruyants à Brighton, mais les regrets (...) étaient passés, et le pavillon royal se montrait plus noir que triste (de Boigne)

"du pavillon royal, il est plus exact de dire qu'il se montrait noir que de dire qu'il se montrait triste", d'où :"plutôt noir que (vraiment) triste"

et de 'parallélisme par anti-symétrie' dans le cas de deux qualités opposées, exprimées par des adjectifs antonymes 'scalaires' (marquant une relation logique de contrariété, càd. d'incompatibilité) ou par des verbes converses (marquant une relation de réciprocité) :

(...) en quoi elle [= la France] était une patrie rationnelle plus qu'une patrie empirique (Ozouf)

Je lui trouve une élégance moins naturelle que recherchée (Crébillon fils)

Je fus moins conduit que traîné vers ma prison (Abbé Prévost)

Les médecins y consentirent plus qu'ils ne m'y encouragèrent (de Boigne)

\subsection{Les cas d'incertitude interprétative}

\subsubsection{La double lecture}

En dehors des cas qui viennent d'être évoqués, la double lecture (standard ou épistémique) est toujours possible. En effet, le parallélisme sémantique ne s'impose plus, dès lors que les qualités, n'étant ni synonymes ni antonymes, n'ont en partage qu'une simple orientation connotative - que celle-ci soit positive ou négative :

Elle était aussi aimable que jolie, pleine de talents et d'esprit (de Boigne)

Comme les hommes sont moins galants que tendres, les femmes sont moins coquettes que sensibles (Rousseau)

Comme l'empereur Alexandre était fort grand et très sourd, quand la femme était petite il se 
tenait courbé, ce qui était plus obligeant que gracieux (de Boigne)

J'avertis seulement qu'elle est moins douloureuse qu'effrayante (Tissot)

Le redressement économique européen semble aussi incertain que lointain (Le Monde)

Plus triste qu'amer face à la pente dangereuse que prend selon lui l'Eglise catholique, le théologien [Hans Küng] ne baisse pas les bras (Le Monde)

Dans des cas de ce type, l'incertitude ne peut être levée que par la présence de marqueurs spécifiques à l'épistémique :

Ardent jusqu'à l'effervescence, susceptible autant que brillant, il était tendu à l'extrême (de Gaulle)

\subsubsection{L'incertitude interprétative}

L'incertitude interprétative, sans doute encore plus massivement attestée en français qu'en anglais (du fait d'un moindre nombre de formes spécifiques à l'épistémique), est une donnée de fait, empiriquement observable sur corpus, et confirmée par la difficulté qu'ont les locuteurs à opérer la distinction entre les deux types de lecture. Plutôt que d'y voir un cas d'ambiguïté dans le sens fort du terme, càd. un choix nécessaire et impossible entre deux solutions mutuellement exclusives, on considèrera (cf. Morycki, sous presse) que les schémas comparatifs non univoques relèvent d'une description en termes d' 'indétermination'. Ce qui revient à dire qu'en l'absence d'indice formel orientant à coup sûr sa lecture, le récepteur peut construire une interprétation qui ne tranche pas entre les deux lectures, ou bien passer insensiblement de l'une à l'autre sans que cela induise de différence notable au plan de la construction référentielle. Ce cas de figure interprétatif tout à fait particulier n'est pas sans rappeler celui des pseudoambiguités du type bonnet de coton blanc, où l'opposition se trouve neutralisée (que l'on choisisse l'un ou l'autre parenthésage).

Considérons en effet les exemples :

J'en fus aussi surprise que désapppointée (de Boigne)

(...) cette chambre où des meubles, plus utiles qu'élégants, deux fois répétés, prouvaient les habitudes les plus conjugales, selon l'usage du pays (de Boigne)

La lecture standard (intraprédicative) fait porter la mesure relative du degré sur les prédicats : “j’en fus surprise à un degré égal à celui auquel j'en fus désappointée", "(meubles) d'un degré d'utilité supérieur à leur degré d'élégance". La lecture épistémique (extraprédicative) la fait porter sur les assertions : "il est aussi exact de dire que j'en fus surprise que de dire que j'en fus désappointée", "(meubles) dont il est plus exact de dire qu'ils sont utiles que de dire qu'ils sont élégants". Or ces deux lectures, qui correspondent bel et bien à deux stratégies différentes (d'où deux types de représentations métalinguistiques distinctes en termes de portée du quantificateur ${ }^{7}$ ) ne conduisent pas, pour autant, à des solutions référentielles mutuellement exclusives. Et l'on peut facilement glisser de l'une à l'autre. A cet égard, ce n'est sans doute pas un hasard si les (nombreux) schémas susceptibles d'une double lecture sont ceux qui exhibent l'ordre des constituants typique de la comparaison standard.

Mais s'en tenir à cette indétermination reviendrait à négliger les différences d'ordre pragmatique, qui touchent le jeu des préconstruits et la dimension argumentative.

\section{Les effets pragmatiques}

La comparaison épistémique est susceptible de donner lieu à trois grands types d'effets, selon qu'elle opère entre les deux assertions une disjonction inclusive, une disjonction exclusive ou une conjonction. 


\subsection{La disjonction inclusive}

Premier cas de figure : l'un des deux contenus propositionnels présente un degré d'adéquation au réel supérieur à l'autre, mais les deux sont donnés comme susceptibles d'être vrais ensemble.

\subsubsection{Les schémas}

On trouve ici les schémas d'inégalité comportant deux qualités A et B quasi-synonymes, càd. participant d'un même domaine notionnel :

C'était de ces visages qui ont l'air plus ancien $(=\mathrm{A})$ que vieux $(=\mathrm{B})$; on dirait que le temps les ménage (...) (Marivaux)

La nature, envers moi moins mère $(=\mathrm{B})$ que marâtre $(=\mathrm{A})$, / M'a formé très rétif et très opiniâtre (...) (Destouches)

Ces deux schémas symétriques reviennent à poser que l'entité n'a "pas vraiment" la qualité B, mais plutôt la qualité A ; autrement dit, le terme A convient davantage que le terme B pour qualifier l'entité, et donc il est plus pertinent d'asserter la proposition contenant A que la proposition contenant B.

On trouve aussi les schémas d'inégalité comportant deux qualités qui relèvent de deux domaines notionnels connexes et de même orientation connotative :

Elle [la loi de 1901 sur les associations] est plus timide que la loi syndicale (...) ; tardive aussi, puisqu'elle consacre plus $(=\mathrm{A})$ qu'elle ne crée $(=\mathrm{B})$, tant ont fleuri depuis deux décennies les groupes de tous ordres (...) (Ozouf)

"elle consacre plutôt qu'elle ne crée" ; "elle ne crée pas vraiment, elle consacre plutôt"

Elle paraissait environnée de tous côtés par des rochers semblables à celui que nous venions de traverser. Ils étaient moins hauts $(=\mathrm{B})$ que raides et escarpés (= A). (Abbé Prévost)

"pas tant hauts que (plutôt) raides et escarpés"; "pas vraiment hauts, mais plutôt raides et escarpés"

On trouve enfin les schémas comportant deux constituants du prédicat qui dénotent deux perspectives non exclusives. Dans les schémas d'inégalité, A est alors donné comme un élément rhématique plus pertinent que B :

La croissance britannique (...) relève davantage de son appartenance au monde angloaméricain (= A) (...) que de la valeur de sa monnaie (= B) (ex. de Bres, 1999)

La spécificité de l'époque actuelle réside moins dans la critique de la police $(=\mathrm{B})$ que dans la façon dont celle-ci est formulée (= A) (ex. de Bres, 1999)

Dans les schémas négatifs d'égalité, l'énoncé équivaut à une inégalité de type infériorité (B est moins pertinent que A) :

\section{Ce n'est pas tant l'euro qui s'apprécie (= B) que le dollar qui se déprécie (= A) (Google)}

Le problème [à propos de l'ENA] n'est pas tant dans le classement $(=\mathrm{B})$ que dans la spécialisation (= A) (Les Echos)

Dans les schémas positifs d'égalité opérant sur 'l'échelle des grandes quantités' (cf. Rivara, 1990 : 74 79), l'énoncé équivaut à la négation d'une relation d'infériorité, et dans ceux qui opèrent sur 'l'échelle des petites quantités', il équivaut à la négation d'une relation de supériorité :

La rhétorique familiale se trouve autant dans la littérature de la Résistance $(=\mathrm{A})$ que dans celle de Vichy (= B) (ex. de Bres, 1999)

"au moins autant (voire plus) A que B"; en tout cas "pas moins A que B"

Il est capable de recoudre un bouton $(=\mathrm{B})$ autant que moi de piloter un avion $(=\mathrm{A})$ 


\subsubsection{L'effet de 'correction adversative'}

Du point de vue argumentatif, ce premier type d'effet peut être qualifié de 'correction adversative'(cf. Orlandini, sous presse, à propos des emplois similaires du quam latin). Le terme B est corrigé par le terme A, jugé plus pertinent par l'énonciateur. Comme le note Abe (1995), à propos du schéma plus $p$ que $q$, «l'examen du contexte dévoile une attente implicite ou explicite à l'égard de $q$; le comparatif épistémique a donc pour fonction de remplacer l'attente par un nouveau terme $p$ considéré comme plus pertinent ». En présentant ainsi l'assertion de la proposition qui contient $\mathrm{A}$ comme plus adéquate que celle de la proposition (préconstruite, attendue, attribuable à autrui) qui contient $\mathrm{B}$, l'énonciateur s'inscrit sous l'argument de plus grande pertinence et attribue à un autre (ou à lui-même dans le cadre d'un dédoublement de point de vue) l'argument de moindre pertinence. C'est "nous avons raison tous les deux, mais j'ai plus raison que toi, tu as moins raison que moi", pour reprendre les termes de Bres (1999: § 3). En cela, il ménage son interlocuteur, contrairement à ce qui se passe dans le cas de la disjonction exclusive.

Cette recherche du terme juste, et donc de l'assertion la plus adéquate, pourrait s'exprimer à l'aide d'une simple coordination :

La statue du Bourgeois, elle qui n'a pas été renversée par le Grand Soir attendu, s'effrite et se délite sous nos yeux, ou plutôt elle fond comme neige au soleil, de jour en jour, et cette fois sans chute annoncée (Jullien)

"elle fond, plus/plutôt qu'elle ne s'effrite et se délite"

\subsection{La disjonction exclusive}

Deuxième cas de figure : l'un des deux contenus propositionnels présente un degré d'adéquation au réel supérieur à l'autre, mais les deux sont donnés comme mutuellement exclusifs.

\subsubsection{Les schémas}

On trouve ici les schémas comportant deux qualités A et B non gradables :

Un bon taille-haie est thermique (= A) plutôt qu'électrique (= B) (Le Monde)

On trouve aussi les schémas d'inégalité comportant deux qualités A et B antonymes (converses ou contraires) :

La bonne humeur a quelque chose de généreux : elle donne (= A) plutôt qu'elle ne reçoit $(=\mathrm{B})$ (Alain)

Il apaise d'abord tous les scrupules, en nous insinuant que tous les systèmes sont fort indifférents à la religion, qu'ils seraient même plus utiles $(=\mathrm{A})$ que nuisibles $(=\mathrm{B})$ (Abbé Baruel)

Du fait du sémantisme opposé des deux qualités, les deux propositions ne peuvent être vraies ensemble. Donc, s'il est plus exact d'asserter la proposition contenant A que la proposition contenant B, c'est qu'il n'est pas exact d'asserter cette dernière : l'entité a bien la qualité A, mais "vraiment pas" la qualité B. Ce qui revient à une exclusion hors du domaine notionnel B ("pas du tout B"), au profit du seul A.

\subsubsection{L'effet de 'réfutation'}

Contrairement aux cas de disjonction inclusive, ici l'énonciateur ne ménage pas son interlocuteur au plan argumentatif. Ce n'est plus "nous avons raison tous les deux, bien qu'à des degrés différents", mais “j'ai raison et tu as tort". Considérons l'exemple : 
Elle s'occupait suffisamment de ses affaires pécuniaires pour ne pas les laisser souffrir. Avec les apparences d'un grand abandon dans ses habitudes journalières, elle avait beaucoup d'ordre dans sa fortune qu'elle a plutôt augmentée que dérangée (de Boigne)

L'auteur fait entendre ici deux points de vue radicalement opposés. A ceux qui penseraient qu'elle a dérangé sa fortune (du fait des apparences d'un grand abandon dans ses habitudes journalières), l'auteur oppose son propre avis, à savoir qu'elle a augmenté sa fortune (affirmation étayée par elle s'occupait suffisamment de ses affaires pécuniaires pour ne pas les laisser souffrir et par elle avait beaucoup d'ordre dans sa fortune). Le point de vue préconstruit, attribuable à autrui, est donc réfuté : "elle n'a pas (du tout) dérangé sa fortune, mais elle l'a (au contraire) augmentée".

Au schéma comparatif pourrait correspondre une simple structure parataxique (ou coordonnée), comme dans l'exemple suivant :

Elle n'était pas rapide $(=\mathrm{B})$, elle était plutôt lente $(=\mathrm{A})(\mathrm{Google})$

ou Elle n'était pas rapide, mais elle était au contraire / bien plutôt lente

"elle était lente, plutôt que rapide"; "elle était lente et non pas rapide"

\subsection{La conjonction}

Troisième cas de figure : les deux contenus propositionnels présentent un égal degré d'adéquation au réel et sont donc donnés comme compatibles.

\subsubsection{Les schémas}

On trouve ici des schémas d'égalité positive :

Il était une fois une princesse aussi belle que bonne (ex. de Anscombre, 1975/76)

Ces façades élégantes voilent en général des masures hideuses où se trouvent des dédales de logements, aussi incommodément distribués sur pauvrement habités (de Boigne)

Gabrielle Witkop nous a légué une œuvre parcimonieuse autant que précieuse, traversée par des thématiques fortes (Le Monde)

Les deux qualités $\mathrm{A}$ et $\mathrm{B}$ sont présentées comme également adéquates pour qualifier l'entité, qui est donc tout à la fois "et A et B", avec une valeur de haut degré dans le cas de aussi ("très A et très B"), si bien que les deux assertions positives sont justifiées.

On trouve aussi des schémas d'inégalité négative. La négation d'une relation d'infériorité équivaut alors à l'égalité sur l'échelle des grandes quantités, et la négation d'une relation de supériorité équivaut à l'égalité sur l'échelle des petites quantités :

C'est une spéculation digne d'occuper tout homme curieux de se pénétrer des vérités utiles; spéculation qui n'est pas moins agréable qu'instructive (Baudeau)

"qui est aussi agréable qu'instructive"; "qui est très agréable et très instructive"

Sans doute, ce n'était pas plus notre but que notre pensée, mais, assurément, il ne fallait pas beaucoup de malveillance pour l'expliquer ainsi (de Boigne)

"c'était aussi peu notre but que notre pensée"; "ce n'était ni notre but ni notre pensée"

\subsubsection{L'effet de 'renchérissement'}

Au plan argumentatif, il n'y a plus ici d'effet adversatif. Les deux points de vue sont compatibles, et la proposition contenant A vient renchérir sur celle qui contient B : "non seulement B (préconstruit), mais encore/de plus A", ou "non seulement pas B, mais encore/de plus pas A non plus". L'énonciateur apporte donc un élément nouveau qui abonde l'orientation argumentative du préconstruit — pour autant que l'on 
sache encore repérer ce qui relève du nouveau et du préconstruit : dans ces schémas d'égalité, eux aussi paraphrasables par une structure coordonnée, les locuteurs se trompent souvent et donnent pour nouveau le contenu de la subordonnée!

\section{Conclusion}

L'étude des trois types d'effets pragmatiques a montré que la lecture épistémique autorise une reformulation des énoncés comparatifs à l'aide de schémas de coordination : "A et/mais pas vraiment B" (disjonction inclusive), "A et/mais vraiment pas B" (disjonction exclusive), "A et B / non seulement B mais aussi A" ou "ni A ni B / non seulement pas B mais aussi pas A" (conjonction). Comme le note Muller (1996 : 138), «les marqueurs de comparaison sont de plus en plus utilisés comme de purs connecteurs structurant le discours (...); dans ce cas, tout l'appareil comparatif est extérieur à l'énoncé proprement dit ».

La notion de degré semble ainsi s'effacer au profit de la seule opposition entre assertions (positives et/ou négatives). On pourrait être tenté de voir là une sorte de dilution de la catégorie de la comparaison. Or il n'en est rien : comme le notait déjà Van Overbeke (1975:139-140), cette réduction des antonymes ou synonymes gradués à une opposition disjonctive ou conjonctive n'est pas sans rappeler la façon dont certaines langues expriment la comparaison standard à l'aide de schémas dits de 'comparatives conjointes'(Stassen, $1985: 44-45$ et 183-188), ou 'schémas de polarité'(Heine, $1997: 116-117)$. Dans ces langues, "A est plus grand que B" se dit $A$ est grand et B est petit (ou $B$ n'est pas grand) et "A est aussi grand que B" se dit $A$ est grand et $B$ est grand (Henkelmann, $2006: 376$ ). Il y a là une stratégie de 'coordination adversative' totalement implicite qui consiste à juxtaposer deux prédications, sans aucun encodage explicite du concept d'inégalité ou d'égalité.

On voit par là que, loin d'opérer une sorte de détournement du schéma comparatif, la comparaison épistémique constitue au contraire un révélateur privilégié des liens étroits qui unissent gradation et assertion. Ce que la comparaison standard ne connait que de façon marginale (via quelques langues) se trouve massivement attesté ici. Rien d'étonnant à cela : il est, en effet, d'autant plus facile d'exprimer une relation de comparaison à l'aide de la seule coordination d'assertions polarisées que le quantificateur de degré opère, non pas au niveau prédicatif, mais au niveau métalinguistique des assertions.

\section{Références bibliographiques}

Abe, H. (1995). About polysemy in the comparative construction. Furansugogaku kenkyu. 29. 38-45.

Amsili, P. (2006). Antonymie. In Godard, D., Roussarie, L. \& Corblin, F. (éds.), Sémanticlopédie: dictionnaire de sémantique, GDR Sémantique \& Modélisation, CNRS, http://www.semantique-gdr.net/dico/

Amsili, P. \& M. Desmets (sous presse). Les comparatives scalaires parenthétiques. Actes du colloque La scalarité dans tous ses états (Gand, déc. 2008).

Anscombre, J-C. (1975 \& 1976). Il était une fois une princesse aussi belle que bonne. Semantikos. 1 \& 2. 1 -28 \& 1 26.

Bres, J. (1999). Discours, textualité et production de sens. Modèles linguistiques. XX:2. 71-86.

Damourette, J. \& E. Pichon (1911-1927). Essai de Grammaire de la Langue Française. Paris : d'Artrey.

Fuchs, C. (sous presse). La comparaison épistémique en français pré-classique et classique. Actes du Colloque Diachro4 (Madrid, 2008). Berne : Peter Lang, 185-202.

Giannakidou, A. \& M. Stavrou (2008). Metalinguistic comparatives in Greek. Workshop on Greek Syntax and Semantics (David Hill, ed.). Cambridge MA : MIT Working Papers in Linguistics.

Giannakidou, A. \& S. Yoon (sous presse). Metalinguistic comparatives in Greek and Korean: ellipsis, attitude semantics, expressive content and npi licensing. International Conference on Elliptical Constructions, The University of Chicago Center in Paris. 
Heine, B. (1997). Cognitive Foundations of Grammar. Oxford : Oxford University Press.

Henkelmann, P. (2006). Constructions of equative comparison. Sprachtypologie und Universalienforschung. $59: 4$. 370-398.

Inkova, O. (sous presse). Scalarité endophrastique vs scalarité exophrastique : quels types d'échelle ? Actes du Colloque La scalarité dans tous ses états (Gand, déc. 2008).

Kennedy, C. (1996). Comparison of deviation. Linguistics Society of America. Chicago.

Klein, E. (1991). Comparatives. In von Stechow, A. \& Wunderlich, D. (éds.) Handbücher zur Sprach- und Kommunikationswissenschaft 6 : Semantics. Berlin/New-York : de Gruyter. 673-691.

Lyons, J. (1977). Semantics, vol. 1. Cambridge : Cambridge University Press.

Martin, R. (1976). Inférence, antonymie et paraphrase. Paris : Klincksieck.

Morycki, M. (sous presse). Metalinguistic comparison in an alternative semantics for imprecision. In Abdurrahman, M. \& al. (eds.) Proceedings of NELS 38. Amherst Mass. : GLSA Publications. https://www.msu.edu/ morzycki//

Muller, C. (1996). La subordination en français ; le schème corrélatif. Paris : Armand Colin.

Orlandini, A. (sous presse). "Autour du latin quam : entre coordination, disjonction et comparaison". In Orlandini, A. \& Poccetti, P. (éds.) Les structures de la coordination en latin et dans les langues de la Méditerranée Ancienne. Louvain/Paris : Peeters (coll. Linguistique).

Rivara, R. (1979). La comparaison quantitative en anglais contemporain. Paris : Champion.

Rivara, R. (1990). Le système de la comparaison. Paris : Minuit.

Sandfeld, K. (1977²). Syntaxe du français contemporain : les subordonnées. Genève : Droz.

Stassen, L. (1985). Comparison and Universal Grammar. Oxford/New-York : Blackwell.

Trésor de la langue française Informatisé. CD-Rom. Nancy : CNRS.

Van Overbeke, M. (1975). Antonymie et gradation. La Linguistique. 11. 135-154.

\footnotetext{
${ }^{1}$ Un troisième type de lecture de ce schéma a été proposé, sur l'anglais, par Kennedy (1996), concernant des exemples où les deux qualités sont de polarité opposée (Francis is as reticent as Hilary is long-winded). Cette lecture, qu'il a appelée 'comparaison de déviation', existe également en français : Un autre, qui avait été aussi bon pour elle que Serge avait été ingrat, était son époux (Ohnet; cit. Sandfeld). Il s'agit, comme pour la lecture standard, d'une comparaison quantitative intrapropositionnelle, mais qui concerne le degré remarquable (haut degré) auquel chaque entité possède l'une des deux qualités, càd. 'dévie' du degré considéré comme normal : "le degré auquel un autre avait été bon pour elle est aussi remarquablement déviant de la norme que l'est le degré auquel Serge avait été ingrat". Ce type de comparaison, qui mériterait une étude spécifique pour le français, ne sera pas pris en compte dans la présente contribution.

${ }^{2}$ Pour un aperçu sur la comparaison épistémique dans d'autres langues (telles que le grec, le coréen ou le russe), voir par exemple Giannakidou \& Stavrou (2008), Giannakidou \& Yoon (sous presse), Inkova (sous presse).

${ }^{3}$ Sur des états de langue plus anciens du français, voir Fuchs (sous presse).

${ }^{4}$ Contrairement à l'anglais, où la comparaison standard refuse la réduction de la subordonnée (The table is longer than it is wide; The door is as/less high as it is wide; * The table is longer than wide; * The door is as/less high as wide), alors que l'épistémique l'exige - ou l'admet, selon les locuteurs (John is more slim than lean; * John is more slim than he is lean); cf. Rivara (1979: 784-787).

${ }^{5}$ Les trois types de relation d'antonymie ont reçu des noms divers: (a) antonymie 'stricte', ou 'scalaire', ou 'contrariété', ou 'incompatibilité', ou 'inversion', ou 'opposition'; (b) antonymie 'polaire', ou 'non gradable', ou 'contradiction', ou 'complémentarité' ; (c) antonymie 'converse', ou 'réciprocité'. Cf. Martin (1976 : 59-75), Lyons (1977 : 279), Amsili (2006).

${ }^{6}$ Dans le cas de prédicats parasynonymes, la difficulté d'avoir un schéma d'égalité s'explique par le fait qu'avec une seule entité, cela conduirait à une quasi-tautologie (??? Elle est aussi jolie que belle) et qu'avec deux entités, le schéma tend à être compris comme une comparaison standard : Son frère Armand, aussi bête que Jules est sot, a
} 
toujours été mené par lui (de Boigne). Dans le cas de prédicats opposés, le schéma d'égalité n'évite l'écueil de la quasi-contradiction qu'à la condition de présenter cette entité comme ambivalente, càd. susceptible de vérifier l'un et l'autre prédicat, selon le point de vue ou la circonstance : Bismark le savait devenu pacifique autant qu'il avait été belliqueux dans sa jeunesse et son âge mûr (Bainville). Mais l'interprétation s'oriente alors vers la 'comparaison de déviation' (cf. supra, note 1), à l'instar des exemples, beaucoup plus courants, où les deux qualités polaires sont prédiquées de deux entités distinctes : La nuit fut aussi douce, aussi calme que la journée avait été chaude et agitée (Barrès ; cit. Damourette \& Pichon).

${ }^{7}$ Notre propos n'étant pas ici d'effectuer une étude de sémantique formelle, mais de faire une description aussi complète que possible des conditions linguistiques de la lecture épistémique, nous ne développerons pas la question des types de représentations métalinguistiques et de modélisations envisageables. 